\title{
Angiopoietin-like protein 2 negatively regulated by microRNA-25 contributes to the malignant progression of colorectal cancer
}

\author{
JUMEI ZHOU, JING WANG, SHENGQI WU, SUYU ZHU, SAI WANG, \\ HUIJUN ZHOU, XIAOQING TIAN, NING TANG and SHAOLIN NIE
}

\begin{abstract}
Department of Gastrointestinal Tumor Surgery, The Affiliated Tumor Hospital of Xiangya Medical School of Central South University, Changsha, Hunan 410013, P.R. China
\end{abstract}

Received April 13, 2014; Accepted July 10, 2014

DOI: $10.3892 / \mathrm{ijmm} .2014 .1909$

\begin{abstract}
Angiopoietin-like protein 2 (ANGPTL2) is associated with tumor progression while dysregulation of its expression has been observed in various types of cancer. However, the expression and role of ANGPTL2 remain exclusive in colorectal cancer (CRC). In the present study, we determined the expression levels of ANGPTL2 in CRC tissues and cells. The roles of ANGPTL2 and miR-25 in the migration and invasion of CRC SW620 and HCT-116 cells were also investigated using transwell assays or scratch wound assays. The results showed that ANGPTL2 increased with metastatic progression. Increased ANGPTL2 and decreased microRNA-25 (miR-25) expression were found to coexist in CRC. The functional studies revealed that knockdown of ANGPTL2 reduced colony formation, and the invasive and migratory abilities of human CRC SW620 and HCT-116 cells. Similarly, overexpression of miR-25 resulted in reduced colony formation, invasion and migration in both cell lines. The overexpression of miR-25 led to a decreased ANGPTL2 mRNA and protein expression, whereas the downregulation of miR-25 resulted in increased ANGPTL 2 mRNA and protein expression, in SW620 and HCT-116 cells. miR-25 directly targeted ANGPTL2 by binding to its 3'-UTR, as determined by the dual luciferase reporter assay. To the best of our knowledge, the results of this study suggest for the first time that the abnormal upregulation of ANGPTL2 in CRC is associated with miR-25 downregulation. Additionally, miR-25-mediated ANGPTL2 promoted the malignant progression of CRC. The present study provides evidence supporting ANGPTL2 and miR-25 as diagnostic or therapeutic targets for CRC.
\end{abstract}

Correspondence to: Professor Shaolin Nie, Department of Gastrointestinal Tumor Surgery, The Affiliated Tumor Hospital of Xiangya Medical School of Central South University, 283 Tongzipo Road, Changsha, Hunan 410013, P.R. China

E-mail: csunieshaolin@163.com

Key words: colorectal cancer, angiopoietin-like protein 2, microRNA-25, metastasis

\section{Introduction}

Colorectal cancer (CRC) is one of the most common malignances and the third lethal cancer with an estimated incidence of one million new cases and a mortality rate of $>600,000$ annually worldwide $(1,2)$. Recent progress in surgical operation combined with chemotherapy for CRC has been beneficial in the early stages of CRC. However, effective treatment for patients with advanced CRC remains unsatisfactory. Metastasis is a critical factor resulting in difficulty in curing cancer. Therefore, studies should be conducted on the therapeutic targets for CRC metastasis.

Angiopoietin is important in angiogenesis and the maintenance of hematopoietic stem cells $(3,4)$. A family of proteins structurally similar to angiopoietin was identified and designated 'angiopoietin-like proteins' (ANGPTLs) (5). It has been well established that ANGPTL2 functions as a chronic inflammatory mediator in obesity (6), atherosclerotic disease (7) and rheumatoid arthritis (RA) (8). Findings of recent studies have shown that ANGPTL2 is commonly upregulated in various types of cancer and plays an oncogenic role in inflammatory carcinogenesis, tumor invasion and metastasis (9-11). Notably, a reduced ANGPTL2 expression induced by methylation surrounding the ANGPTL2 CpG-island and an anti-oncogene role in a stage-dependent manner were observed in ovarian cancer (12). These findings suggested that the specific role of ANGPTL2 in cancer may differ depending on cancer type or stage. Thus, the expression and role of ANGPTL2 in CRC remain unclear.

microRNAs (miRs) have been shown to regulate the expression of a variety of genes pivotal for tumor development and highlight a novel mechanism participating in CRC pathogenesis $(13,14)$. A recent study demonstrated that miR-25 functions in various types of cancer including CRC (15). ANGPTL2 may be a direct target gene of miR-25 as predicted by bioinformatical analysis. However, whether the targeted relationship is established in CRC as well as the consequent functional influence remains unclear.

In this study, we determined the expression levels of ANGPTL2 in CRC tissues and cells. The roles of ANGPTL2 and miR-25 in the migration and invasion of CRC SW620 and HCT-116 cells were investigated using transwell assays and scratch wound assays. The results showed that ANGPTL2 
upregulation induced by miR-25 downregulation contributes to the malignant progression of CRC. Accordingly, we suggest that ANGPTL2 and miR-25 may serve as novel diagnostic or therapeutic targets for CRC metastasis.

\section{Materials and methods}

Cell culture. The protocols for this study were approved by the Ethics Committee of Central South University. CRC HT-29, SW480, SW620, and HCT-116 cell lines as well as human colonic epithelial cells (HCEpic) were obtained from the China Center for Type Culture Collection, Wuhan, China. Cells were cultured in DMEM supplemented with $10 \%$ fetal bovine serum (FBS), $100 \mathrm{IU} / \mathrm{ml}$ penicillin, and $100 \mu \mathrm{g} / \mathrm{ml}$ streptomycin sulfate at $37^{\circ} \mathrm{C}$ in a humidified incubator containing $5 \% \mathrm{CO}_{2}$. The ANGPTL2 siRNA, pre-miR-25, pre-miR-con, anti-miR-25 or anti-miR-con (Fulengen Co., Ltd., Guangzhou, China) were transfected into SW620 and HCT-116 cells using Lipofectamine 2000 (Life Technologies, Carlsbad, CA, USA), according to the manufacturer's instructions.

Quantitative RT-PCR (RT-qPCR). Total RNA was extracted from the cells using TRIzol reagent (Life Technologies) following the manufacturer's instructions. The relative expression of miR-25 was determined using RT-qPCR using a mirVana $^{\mathrm{TM}}$ qRT-PCR microRNA Detection kit (Life Technologies) according to the manufacturer's instructions. Specific primer sets for miR-25 and U6 (used as an internal reference) were obtained from Life Technologies. ANGPTL 2 mRNA expression was detected RT-qPCR using the standard SYBR-Green RT-PCR kit (Takara Bio Inc., Otsu, Japan) as per the manufacturer's instructions. The specific primer pairs used were: ANGPTL2 sense, 5'-GCCACCAAGTGTCAGCCTCA-3' and antisense, 5'-TGGACAGTACCAAACATCCAACATC-3'; $\beta$-actin as an internal sense, 5'-AGGGGCCGGACTCGTCATACT-3' and antisense, 5'-GGCGGCACCACCATGTACCCT-3'. The relative expression of ANGPTL2 mRNA or miR-25 was quantified using GraphPad Prism 4.0 software (GraphPad Software, La Jolla, CA, USA) and the $2^{-\Delta \Delta C t}$ method (16).

Immunohistochemical analysis. A human colon adenocarcinoma tissue microarray (Auragene Bioscience Co., Changsha, China), containing 50 cases (including 9 cases of T2, 16 cases of T3, and 25 cases of T4) of colon adenocarcinoma tissues as well as their matched adjacent normal colon tissues, duplicate scores per case, was processed for immunohistochemical analysis using anti-ANGPTL2 antibody (Millipore, Billerica, MA, USA) as described previously (17). The mean optical density value (D) and area (A) of brown particles in three visual fields of each section were calculated by the Leica Q550 image analysis system (Leica Co., Solms, Germany). The expression levels of ANGPTL2 in tissues were evaluated using the formula: integral density $=\mathrm{D} \times \mathrm{A}$.

Cell invasion assay. The cell invasive ability was estimated using a Cell Invasion Assay kit (Chemicon International, Inc., Temecula, CA, USA) according to the manufacturer's instruction as described previously (18). Briefly, the cells were placed in the upper compartment of the chambers, and RPMI-1640 containing $10 \%$ FBS was added in the lower chambers. After $24 \mathrm{~h}$ of incubation at $37^{\circ} \mathrm{C}$, the cells on the upper face of the membrane were scraped using a cotton swab and cells on the lower face were fixed, stained and observed under a microscope (AE31; Motic Group Co., Ltd., Xiamen, China). The dye on the membrane was dissolved with $10 \%$ acetic acid and dispensed into 96 -well plates $(150 \mu \mathrm{l} /$ well $)$. The optical density at $570 \mathrm{~nm}$ (OD570) of each well was subsequently measured with an ELISA reader (ELX-800 type; BioTek, Winooski, VT, USA).

Cell migration assay. Cell migratory abilitiy was estimated using a wound healing assay as described previously (19). In brief, cells transfected with miRs or its inhibitor were cultured to confluence. Wounds of $\sim 1 \mathrm{~mm}$ width were created with a plastic scriber, and cells were washed and incubated in a serum-free medium. After wounding for $24 \mathrm{~h}$, the cells were incubated in a medium including $10 \%$ FBS. Cultures at 0, 24 and $48 \mathrm{~h}$ were fixed and observed under a microscope.

Western blotting. Total protein was extracted and the protein concentration was measured by the Bradford DC protein assay (Bio-Rad, Hercules, CA, USA). Proteins were then separated in $10 \%$ SDS-PAGE and blotted onto a polyvinylidene difluoride (PVDF) membrane. The PVDF membrane was treated with TBST containing $50 \mathrm{~g} / \mathrm{l}$ skimmed milk at room temperature for $4 \mathrm{~h}$, followed by incubation with the primary antibodies anti-ANGPTL2 and anti- $\beta$-actin (both from Santa Cruz Biotechnology, Inc., Dallas, TX, USA), respectively, at $37^{\circ} \mathrm{C}$ for $1 \mathrm{~h}$. The membranes were rinsed and incubated for $1 \mathrm{~h}$ with the correspondent peroxidase-conjugated secondary antibodies. Chemiluminent detection was performed using an ECL kit (Pierce Chemical Co., Rockford, IL, USA).

Dual luciferase reporter assay. HCT-116 cells were co-transfected with the reporter constructs ANGPTL2-3' UTRpsi-CHECK2 (containing the 3'-UTR of ANGPTL2 including the miRNA-25 binding sites) or Mut-ANGPTL2-3' UTR-psiCHECK2 (containing the corresponding mutated sequence of 3' UTR of ANGPTL2), and miR-25 mimics or negative control (Life Technologies) using Lipofectamine 2000. Luciferase activity was determined after $48 \mathrm{~h}$ using the Dual-Glo substrate system (Promega, Madison, WI, USA) and LD400 Luminometer (Beckman Coulter, Brea, CA, USA). Data were presented as the ratio of experimental (Renilla) luciferase to control (Firefly) luciferase.

Statistical analysis. Data were expressed as the mean \pm standard deviation (SD). Differences between the two groups were determined using a Student's t-test. Analyses were performed using SPSS 16.0 software. $\mathrm{P}<0.05$ was considered statistically significant.

\section{Result}

Expression of ANGPTL2 and miR-25 in CRC tissues and cells. To investigate the expression of ANGPTL2 protein in CRC tissues, a CRC tissue microarray was used. Fig. 1A and B showed that ANGPTL2 was highly expressed in CRC tissues compared with normal tissues, and gradually increased with the metastatic progression of CRC. The levels 
A

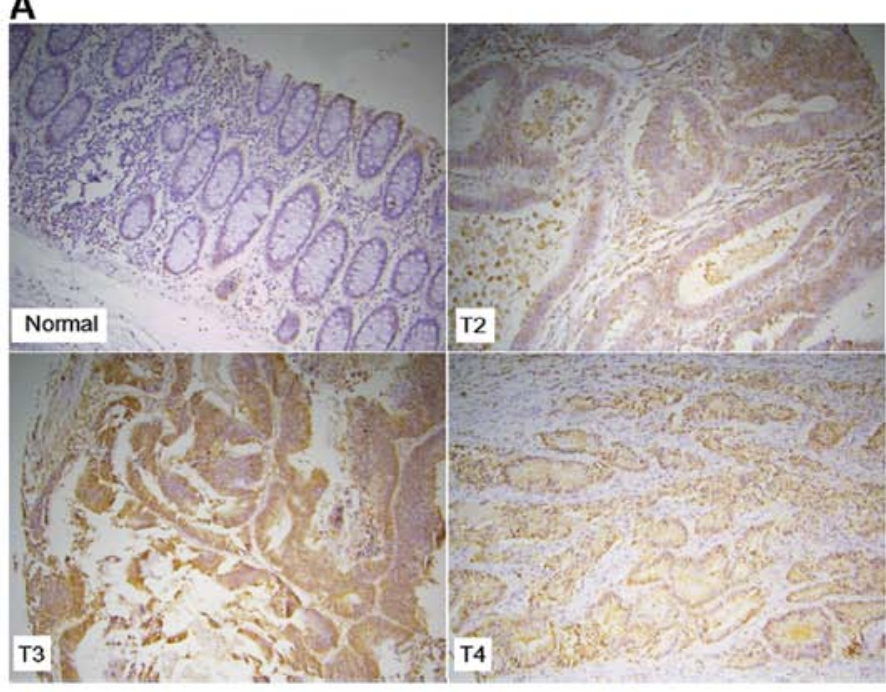

B

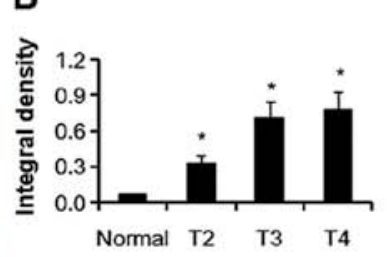

D

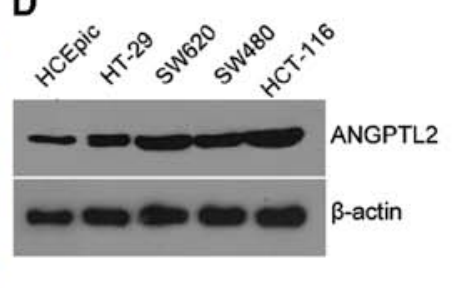

C

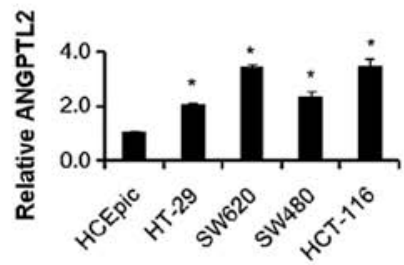

E

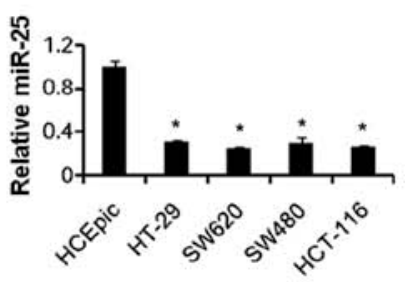

Figure 1. Expression of angiopoietin-like protein 2 (ANGPTL2) and microRNA-25 (miR-25) in colorectal cancer (CRC) tissues and cells. (A) The representative results of ANGPTL2 expression (brown particles) in human colon adenocarcinoma tissue. (B) The ANGPTL2 protein expression (presented as integral density) increased in grading T2, T3 and T4 of colon adenocarcinoma tissues, compared to matched adjacent normal colon tissue (normal), using a tissue microarray by immunohistochemical analysis. Relative expression of ANGPTL2 mRNA (C) and protein (D) in CRC cells. (E) Relative expression of miR-25 in CRC cells. * $\mathrm{P}<0.05$ vs. normal (B) or human colonic epithelial cell (HCEpic) group (C and E).

of ANGPTL2 protein and mRNA expression in CRC HT-29, SW480, SW620, HCT-116 cells and HCEpic were determined using RT-qPCR. In contrast to HCEpic, the ANGPTL2 expression levels were elevated in the CRC cells (Fig. 1C and D). Notably, the RT-qPCR results showed that the miR-25 expression levels in the four CRC cell lines were significantly decreased compared with the control (Fig. 1E). These results suggested that ANGPTL2 likely plays an important role in the malignant progression of CRC. Additionally, the coexistence of ANGPTL2 upregulation and miR-25 downregulation in CRC cells suggests a mutual potential regulatory correlation.

Roles of ANGPTL2 in CRC cells. To investigate the functions of ANGPTL2 in CRC, SW620 and HCT-116 cells were transfected with ANGPTL2 siRNA. Fig. 2A and B shows that the ANGPTL2 mRNA and protein expression of the two cell lines was significantly decreased, indicating that ANGPTL2 was downregulated in SW620 and HCT-116 cells. Knockdown of ANGPTL2 reduced cell colony formation, and the invasive and migratory abilities of SW620 and HCT-116 cells (Figs. 2C-E and 3). Inhibition of ANGPTL2 expression suppressed the invasion and migration of CRC cells. This result suggested that ANGPTL2 plays a promotional role in $\mathrm{CRC}$ metastatic progression.

Roles of miR-25 in CRC cells. To verify the biological roles of miR-25 in CRC, SW620 and HCT-116 cells were transfected with pre-miR-25 or miR-25 inhibitor. Fig. 4A shows that the induction of pre-miR-25 significantly increased miR-25 expression in SW620 and HCT-116 cells. Induction of miR-25 inhibitor significantly decreased its expression. It was then confirmed that the overexpression of miR-25 reduced colony formation, and the invasive and migratory abilities of SW620 and HCT-116 cells. However, inhibition of miR-25 increased proliferation, invasion and migration of the two cell lines (Figs. 4B-D and 5). This result suggested that miR-25 functions as an anti-oncogene in CRC.
Regulation of ANGPTL2 expression by miR-25. To examine the effects of miR-25 on ANGPTL2 expression, the mRNA and protein levels of ANGPTL2 in SW620 and HCT-116 cells transfected with pre-miR-25 or miR-25 inhibitor were determined using RT-qPCR and western blotting. The results showed that the overexpression of miR-25 significantly decreased ANGPTL2 expression in SW620 and HCT-116 cells. By contrast, the downregulation of miR-25 significantly increased ANGPTL2 expression in the two cell lines (Fig. 6A-C). This result demonstrated that miR-25 negatively affected ANGPTL2 expression in CRC cells.

To investigate whether ANGPTL2 is a direct target of miR-25, the ANGPTL2 3'-UTR fragment containing the miR-25 binding site and mutated targeting sequence were subcloned into psi-CHECK2 dual luciferase reporter vectors. Ectopic expression of miR-25 significantly reduced the luciferase activity in HCT-116 cells transfected with the ANGPTL2-3' UTR-psi-CHECK2 reporter vector (Fig. 6D). The luciferase activity levels in HCT-116 transfected with Mut-ANGPTL2-3' UTR-psi-CHECK2 reporter vector plus miR-25 were not significantly different from those of the control. It was confirmed that miR-25 directly targets ANGPTL2. The above results suggested that ANGPTL2 upregulation partly induced by miR-25 downregulation promotes the malignant progression of CRC.

\section{Discussion}

It has been well-established that the initiation and progression of cancers involve the deregulation of various genes, such as oncogene upregulation, anti-oncogene downregulation or loss (20-23). ANGPTL2 expression is reported to be upregulated in lymph node metastasis-positive lung cancer tissues compared with lymph node metastasis-negative cases (11). A high ANGPTL2 expression in lung cancer suggests a poor prognosis in terms of disease-free survival (10). Results of 

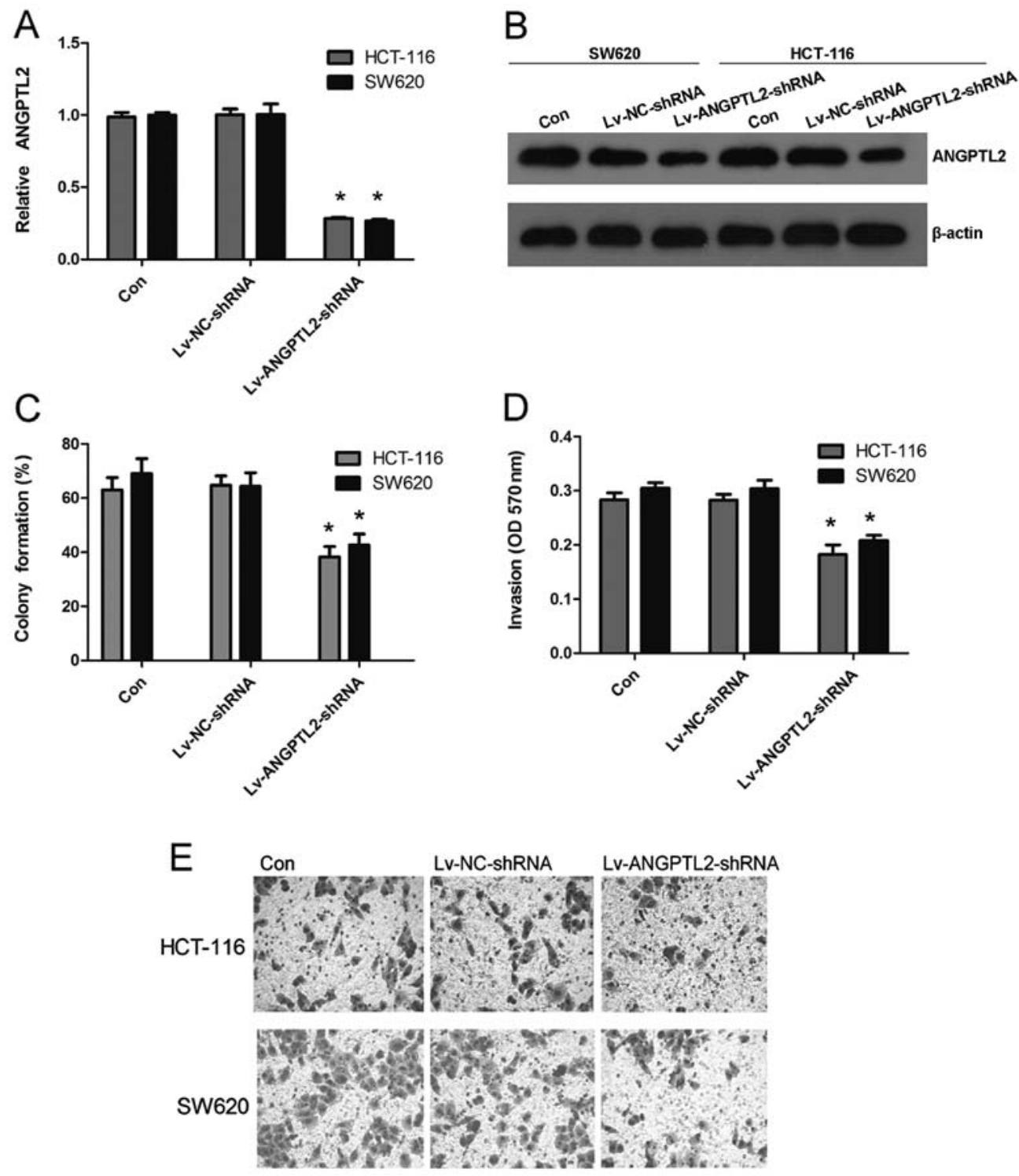

Figure 2. Roles of angiopoietin-like protein 2 (ANGPTL2) in colorectal cancer (CRC) cells. Effects of ANGPTL2 shRNA on (A) mRNA and (B) protein expression of ANGPTL2 in HCT-116 and SW620 cells detected by RT-qPCR or western blotting. Decreased (C) colony formation, and (D and E) invasion in HCT-116 and SW620 cells transfected with ANGPTL2 shRNA. ${ }^{*} \mathrm{P}<0.05$ vs. control (con).
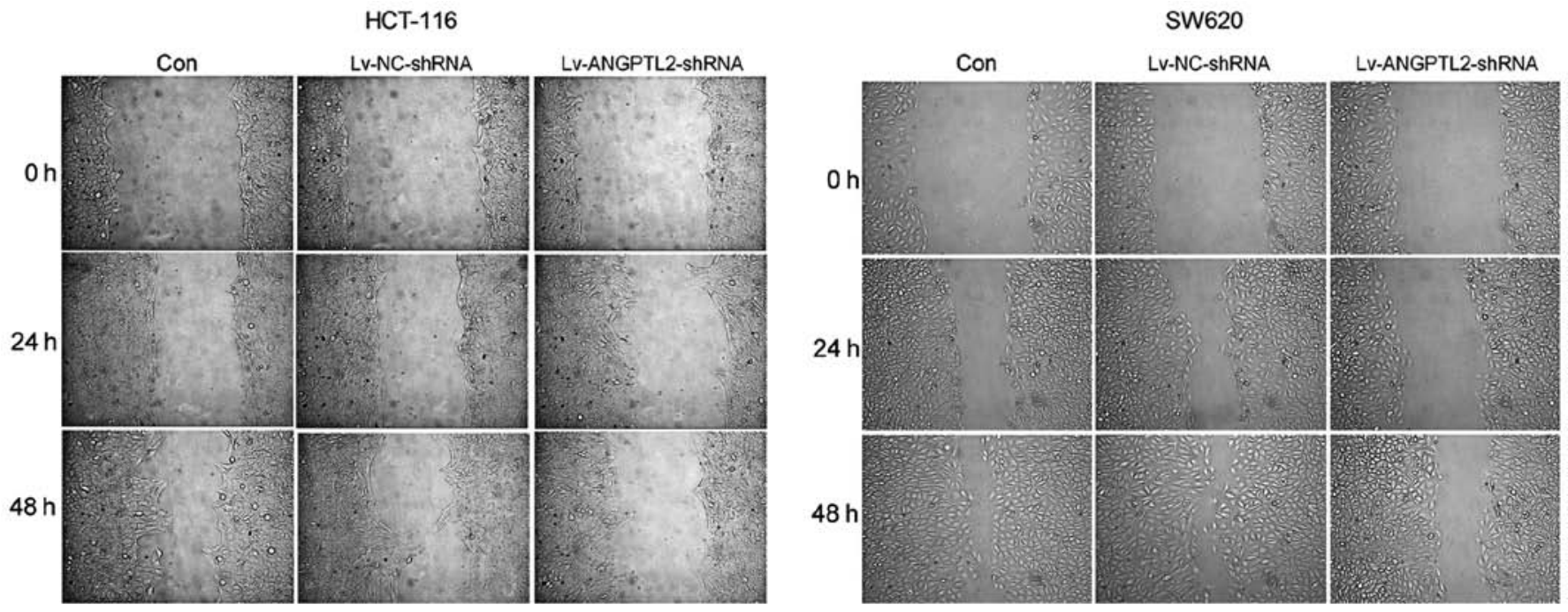

Figure 3. Effects of angiopoietin-like protein 2 (ANGPTL2) siRNA on the migration of colorectal cancer (CRC) cells. The migration decreased in HCT-116 and SW620 cells transfected with ANGPTL2 shRNA using a wound healing assay. 

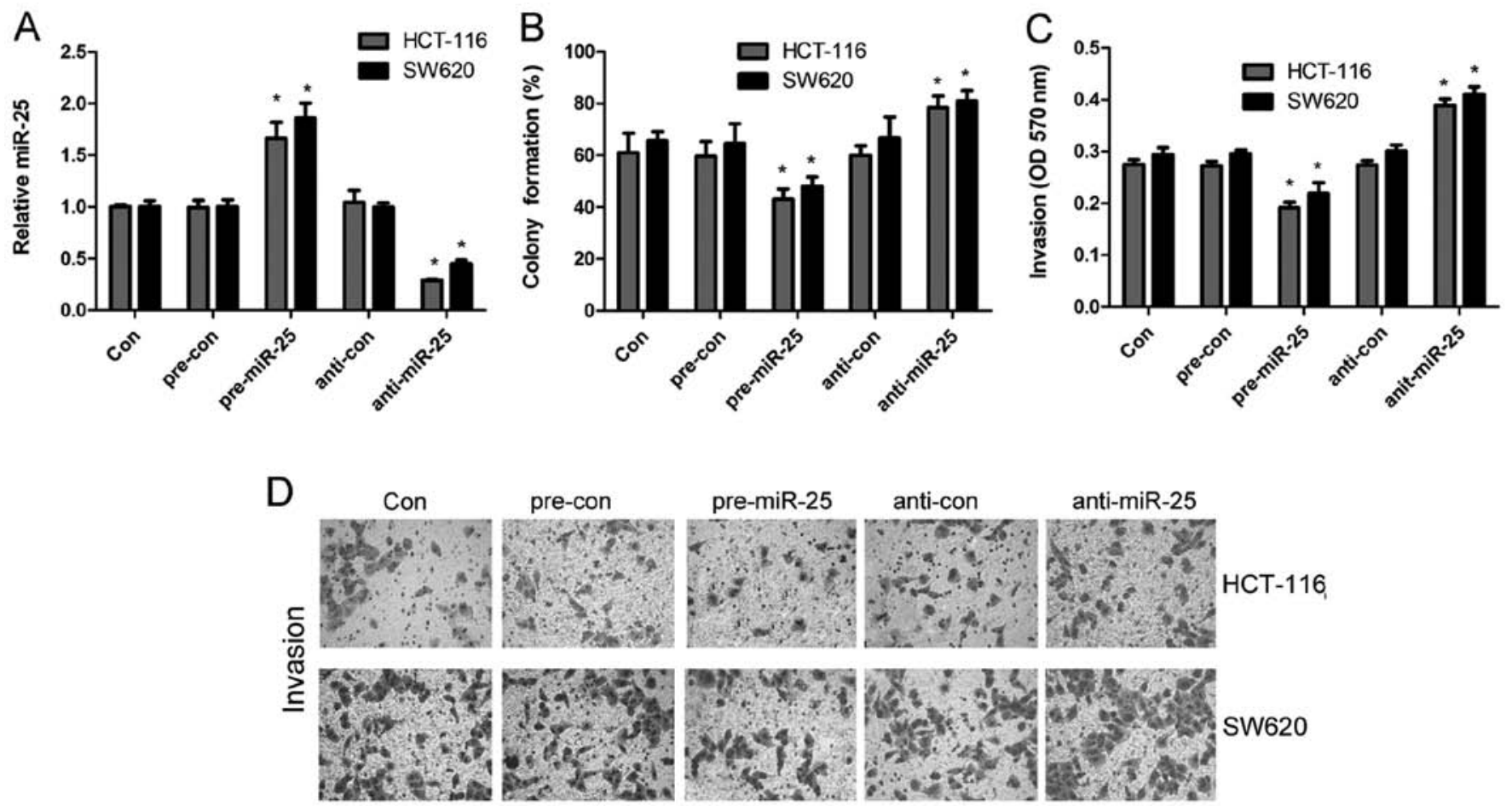

Figure 4. Roles of microRNA-25 (miR-25) in colorectal cancer (CRC) cells. (A) Effects of pre-miR-25 and anti-miR-25 on the expression of miR-25 in HCT-116 and SW620 cells were detected by RT-qPCR. The change of (B) colony formation and (C and D) invasion were examined in HCT-116 and SW620 cells transfected with pre-miR- 25 or anti-miR-25, respectively. ${ }^{*} \mathrm{P}<0.05$ vs. control (con).

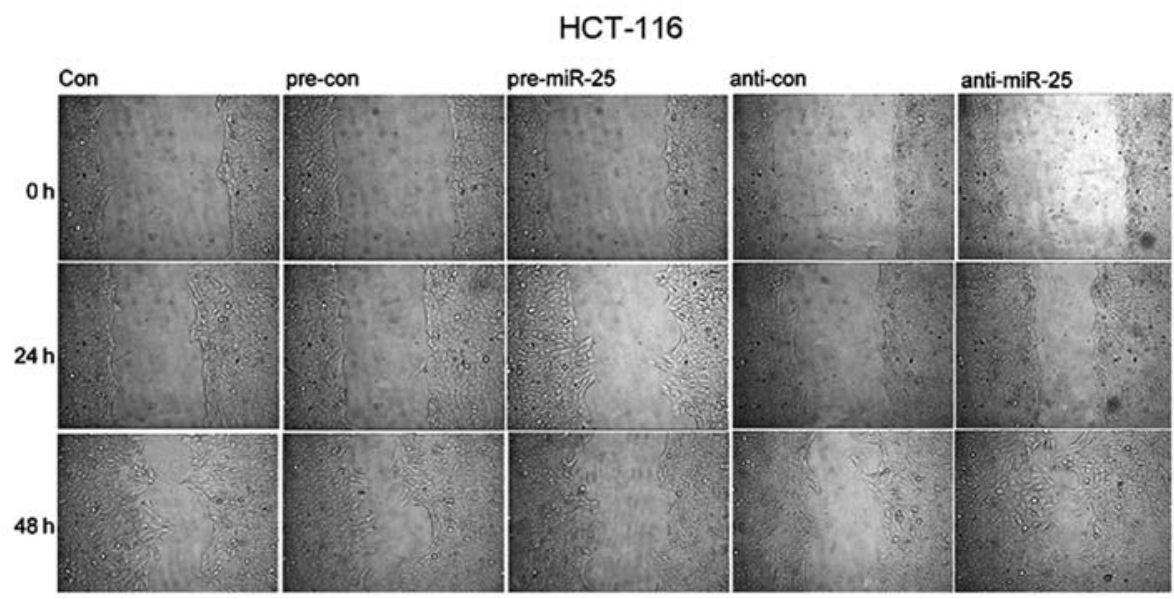

SW620

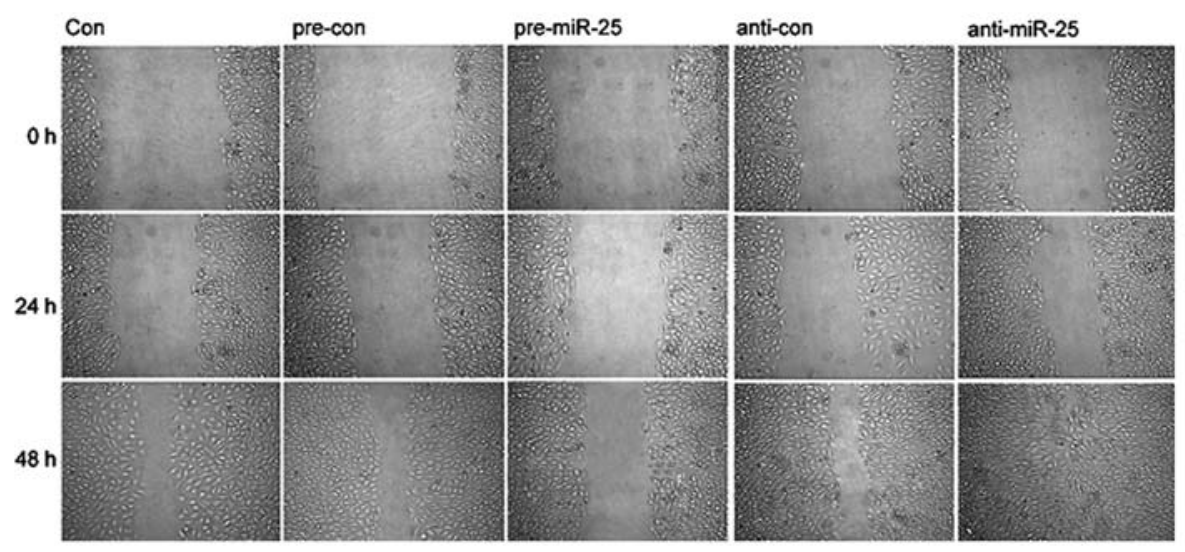

Figure 5. Effects of microRNA-25 (miR-25) on the migration of colorectal cancer (CRC) cells. The migration decreased in HCT-116 and SW620 cells transfected with pre-miR-25, and increased in cells transfected with anti-miR-25, using a wound healing assay. 


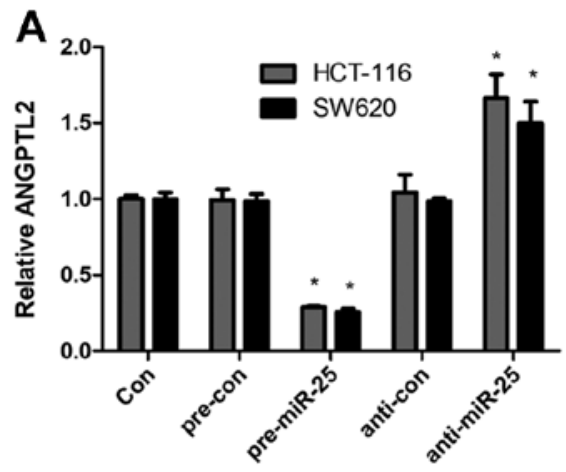

C

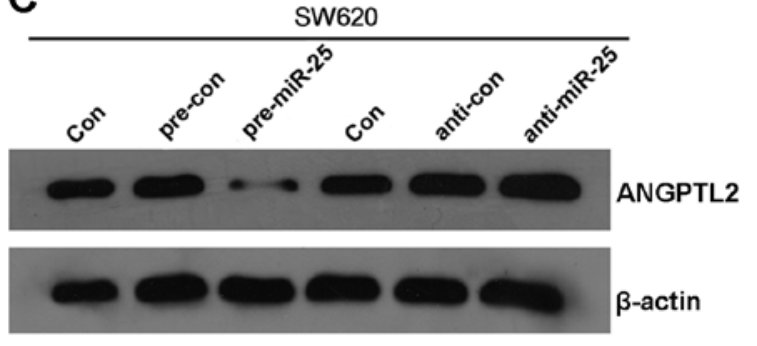

B
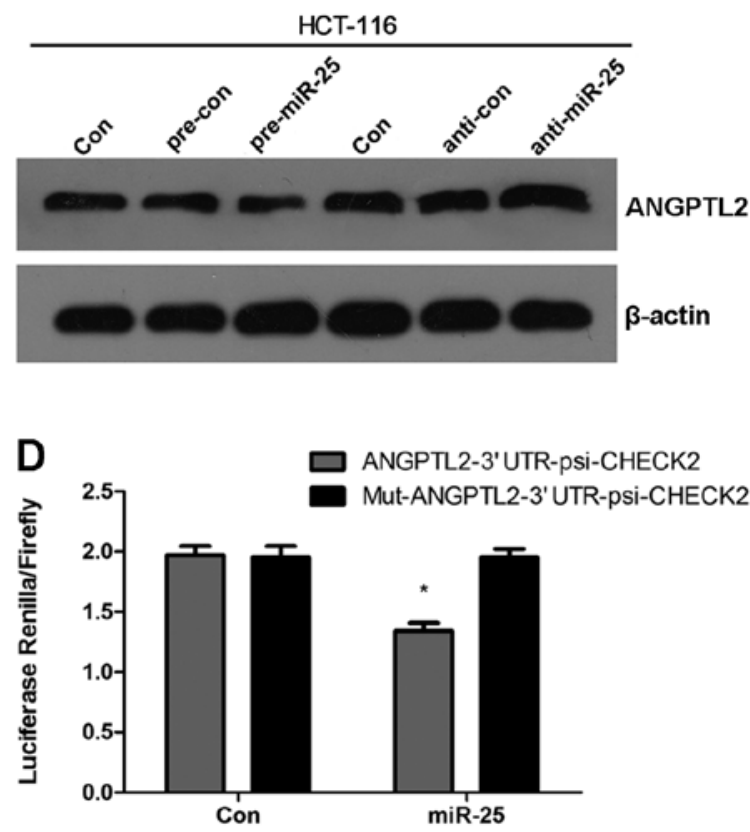

Figure 6. Regulation of angiopoietin-like protein 2 (ANGPTL2) expression by microRNA-25 (miR-25). Effects of pre-miR-25 and anti-miR-25 on (A) mRNA and (B and C) protein expression of ANGPTL2 in HCT-116 and SW620 cells detected by RT-qPCR or western blotting. (D) Dual luciferase reporter assays were performed to examine the interaction of miR-25 and its targeting sequence in the ANGPTL2 3' UTR using constructs containing the targeting sequence (ANGPTL2-3' UTR-psi-CHECK2 reporter vector) and mutated targeting sequence (Mut-ANGPTL2-3' UTR-psi-CHECK2 reporter vector) in HCT-116 cells. ${ }^{*} \mathrm{P}<0.05$ vs. control (con).

the present study show that ANGPTL2 expression was higher in CRC tissues than in matched adjacent normal colon tissue, and gradually increased with metastatic progression. The levels of ANGPTL2 protein and mRNA expression were also elevated in CRC HT-29, SW480, SW620 and HCT-116 cells as compared to HCEpic. These findings suggest that ANGPTL2 is involved in the malignant progression of CRC. We further showed that the knockdown of ANGPTL2 reduced colony formation, and the invasive and migratory abilities of SW620 and HCT-116 cells, suggesting that ANGPTL2 acts as an oncogene in CRC and its upregulation may promote its metastatic progression.

ANGPTL2 upregulation can enhance distant metastasis of cancer possibly through increased tumor angiogenesis depending on Rac activation, and tumor cell epithelial-tomesenchymal transitions (EMT) (9), and can increase in vitro motility and invasion of cancer cells in an autocrine/paracrine manner (10). It has been demonstrated that ANGPTL2 upregulation is associated with increased transcription factors NFATc, ATF2, and c-Jun expression, which form a complex and bind to the ANGPTL2 promoter region (10). However, increasing evidence suggests that the deregulation of tumor-associated genes is partly due to the abnormal expression of its regulatory miRs (24-26). In this study, we examined the expression of miR-25 in CRC cells, which is a theoretic regulatory miR of ANGPTL2 predicted by algorithms available. Our data show that the miR-25 expression levels in the four CRC cell lines were significantly decreased compared to the control, which is consistent with findings obtained by Li et al (15) who found that miR-25 was downregulated in human CRC tissues. Overexpression of miR-25 inhibited colony formation, and the invasive and migratory abilities of SW620 and HCT-116 cells, while the inhibition of miR-25 promoted the invasion and migration of the two cell lines, suggesting that miR-25 contributes to the development of CRC, possibly by targeting ANGPTL2.

An inverse correlation between the expression of miR-25 and the ANGPTL2 mRNA and protein expression levels in CRC cells was identified, suggesting a critical role of miR-25 in the regulation of ANGPTL2 expression by targeting its 3'-UTR. Thus, ANGPTL2 upregulation in CRC is partly due to a decreased miR-25 expression. The regulatory mechanisms of the tumor-associated gene are complicated, involving methylation $(27,28)$, mutation (29), transcription factors (30) and miRs (31). In CRC, an increased ANGPTL2 expression is also possibly associated with NFATc, ATF2, and c-Jun as well as in lung cancer (10), or the other potential miRs which may target ANGPTL2. A gene can be regulated by multiple miRs and a miR may also target multiple genes $(32,33)$. Recently, it has been reported that miR-25 is capable of targeting Smad7 leading to inhibition of the proliferation and migratory ability of CRC cells (15), and target the polycomb protein enhancer of zeste $2(\mathrm{EZH} 2)$ resulting in inhibition of proliferation and colony formation of anaplastic thyroid carcinoma cells by inducing G2/M-phase cell-cycle arrest (34). In addition, molecules involved in the regulation of proliferation, apoptosis, cell cycle, migration, invasion, and other biological process, are also targets of miR-25 in various types of cell, such as the TNF-related apoptosis inducing ligand (TRAIL) death receptor-4 (35), Bim (36), Scratch2 (37), Wwp2, Fbxw7 (38) and MITF (39). Whether these genes regulated by miR-25 play a role in CRC should be investigated. 
In summary, the ANGPTL2 is upregulated in CRC and gradually increases with its metastatic progression. The upregulation of ANGPTL2 may be partly induced by miR-25 downregulation and miR-25 targeting ANGPTL2 contributes to the metastatic progression of CRC. Our results provide evidence supporting miR-25 and ANGPTL2 as diagnostic or therapeutic tools for CRC.

\section{References}

1. Parkin DM, Bray F, Ferlay J and Pisani P: Global cancer statistics, 2002. CA Cancer J Clin 55: 74-108, 2005.

2. Jemal A, Bray F, Center MM, Ferlay J, Ward E and Forman D: Global cancer statistics. CA Cancer J Clin 61: 69-90, 2011.

3. Suda T, Arai F and Shimmura S: Regulation of stem cells in the niche. Cornea 24: S12-S17, 2005.

4. Yancopoulos GD, Davis S, Gale NW, Rudge JS, Wiegand SJ and Holash J: Vascular-specific growth factors and blood vesse formation. Nature 407: 242-248, 2000.

5. Hato T, Tabata M and Oike Y: The role of angiopoietin-like proteins in angiogenesis and metabolism. Trends Cardiovasc Med 18: 6-14, 2008

6. Tabata M, Kadomatsu T, Fukuhara S, et al: Angiopoietin-like protein 2 promotes chronic adipose tissue inflammation and obesity-related systemic insulin resistance. Cell Metab 10: 178-188, 2009.

7. Tazume H, Miyata K, Tian Z, et al: Macrophage-derived angiopoietin-like protein 2 accelerates development of abdominal aortic aneurysm. Arterioscler Thromb Vasc Biol 32: 1400-1409, 2012.

8. Okada T, Tsukano H, Endo M, et al: Synoviocyte-derived angiopoietin-like protein 2 contributes to synovial chronic inflammation in rheumatoid arthritis. Am J Pathol 176 2309-2319, 2010.

9. Aoi J, Endo M, Kadomatsu T, et al: Angiopoietin-like protein 2 is an important facilitator of inflammatory carcinogenesis and metastasis. Cancer Res 71: 7502-7512, 2011.

10. Endo M, Nakano M, Kadomatsu T, et al: Tumor cell-derived angiopoietin-like protein ANGPTL2 is a critical driver of metastasis. Cancer Res 72: 1784-1794, 2012.

11. Sasaki H, Suzuki A, Shitara M, et al: Angiopoietin-like protein ANGPTL2 gene expression is correlated with lymph node metastasis in lung cancer. Oncol Lett 4: 1325-1328, 2012.

12. Kikuchi R, Tsuda H, Kozaki K, et al: Frequent inactivation of a putative tumor suppressor, angiopoietin-like protein 2 , in ovarian cancer. Cancer Res 68: 5067-5075, 2008.

13. Schetter AJ and Harris CC: Alterations of microRNAs contribute to colon carcinogenesis. Semin Oncol 38: 734-742, 2011.

14. Wu WK, Law PT, Lee CW, et al: MicroRNA in colorectal cancer: from benchtop to bedside. Carcinogenesis 32: 247-253, 2011.

15. Li Q, Zou C, Zou C, et al: MicroRNA-25 functions as a potential tumor suppressor in colon cancer by targeting Smad7. Cancer Lett 335: 168-174, 2013.

16. Arocho A, Chen B, Ladanyi M and Pan Q: Validation of the 2-DeltaDeltaCt calculation as an alternate method of data analysis for quantitative PCR of BCR-ABL P210 transcripts. Diagn Mol Pathol 15: 56-61, 2006.

17. Jiang X, Yue J, Lu H, et al: Inhibition of filamin-A reduces cancer metastatic potential. Int J Biol Sci 9: 67-77, 2013.

18. Aspenström P, Fransson A and Saras J: Rho GTPases have diverse effects on the organization of the actin filament system. Biochem J 377: 327-337, 2004.

19. Saadoun S, Papadopoulos MC, Hara-Chikuma M and Verkman AS: Impairment of angiogenesis and cell migration by targeted aquaporin-1 gene disruption. Nature 434: 786-792, 2005 .
20. Lai AZ, Durrant M, Zuo D, Ratcliffe CD and Park M: Met kinase-dependent loss of the $\mathrm{E} 3$ ligase $\mathrm{Cbl}$ in gastric cancer. $\mathrm{J}$ Biol Chem 287: 8048-8059, 2012.

21. Baldwin A, Huh KW and Munger K: Human papillomavirus E7 oncoprotein dysregulates steroid receptor coactivator 1 localization and function. J Virol 80: 6669-6677, 2006.

22. Hill R, Calvopina JH, Kim C, et al: PTEN loss accelerates KrasG12D-induced pancreatic cancer development. Cancer Res 70: 7114-7124, 2010.

23. Woods NT, Yamaguchi H, Lee FY, Bhalla KN and Wang HG: Anoikis, initiated by Mcl-1 degradation and Bim induction, is deregulated during oncogenesis. Cancer Res 67: 10744-10752, 2007.

24. Kadera BE, Li L, Toste PA, et al: MicroRNA-21 in pancreatic ductal adenocarcinoma tumor-associated fibroblasts promotes metastasis. PLoS One 8: e71978, 2013.

25. Dahlhaus M, Schult C, Lange S, Freund M and Junghanss C: MicroRNA 181a influences the expression of HMGB1 and CD4 in acute Leukemias. Anticancer Res 33: 445-452, 2013.

26. Sandhu R, Rivenbark AG and Coleman WB: Loss of post-transcriptional regulation of $D N M T 3 b$ by microRNAs: A possible molecular mechanism for the hypermethylation defect observed in a subset of breast cancer cell lines. Int J Oncol 41: 721-732, 2012.

27. Fang JY, Lu J, Chen YX and Yang L: Effects of DNA methylation on expression of tumor suppressor genes and proto-oncogene in human colon cancer cell lines. World J Gastroenterol 9: 1976-1980, 2003.

28. Pakneshan P, Szyf M and Rabbani SA: Methylation and inhibition of expression of uPA by the RAS oncogene: divergence of growth control and invasion in breast cancer cells. Carcinogenesis 26 : 557-564, 2005

29. Chien $\mathrm{CH}$ and Chow SN: Point mutation of the ras oncogene in human ovarian cancer. DNA Cell Biol 12: 623-627, 1993.

30. Beger M, Butz K, Denk C, Williams T, Hurst HC and Hoppe-Seyler F: Expression pattern of AP-2 transcription factors in cervical cancer cells and analysis of their influence on human papillomavirus oncogene transcription. J Mol Med (Berl) 79: 314-320, 2001.

31. Patel JB, Appaiah HN, Burnett RM, et al: Control of EVI-1 oncogene expression in metastatic breast cancer cells through microRNA miR-22. Oncogene 30: 1290-1301, 2011.

32. Shenouda SK and Alahari SK: MicroRNA function in cancer: oncogene or a tumor suppressor? Cancer Metastasis Rev 28: 369-378, 2009.

33. Lukiw WJ and Alexandrov PN: Regulation of complement factor $\mathrm{H}(\mathrm{CFH})$ by multiple miRNAs in Alzheimer's disease (AD) brain. Mol Neurobiol 46: 11-19, 2012.

34. Esposito F, Tornincasa M, Pallante P, et al: Down-regulation of the miR-25 and miR-30d contributes to the development of anaplastic thyroid carcinoma targeting the polycomb protein EZH2. J Clin Endocrinol Metab 97: E710-E718, 2012.

35. Razumilava N, Bronk SF, Smoot RL, et al: miR-25 targets TNF-related apoptosis inducing ligand (TRAIL) death receptor-4 and promotes apoptosis resistance in cholangiocarcinoma. Hepatology 55: 465-475, 2012.

36. Zhang H, Zuo Z, Lu X, Wang L, Wang H and Zhu Z: miR-25 regulates apoptosis by targeting Bim in human ovarian cancer. Oncol Rep 27: 594-598, 2012.

37. Rodríguez-Aznar E, Barrallo-Gimeno A and Nieto MA: Scratch2 prevents cell cycle re-entry by repressing miR-25 in postmitotic primary neurons. J Neurosci 33: 5095-5105, 2013.

38. Lu D, Davis MP, Abreu-Goodger C, et al: miR-25 regulates Wwp2 and Fbxw7 and promotes reprogramming of mouse fibroblast cells to iPSCs. PLoS One 7: e40938, 2012.

39. Zhu Z, He J, Jia X, et al: MicroRNA-25 functions in regulation of pigmentation by targeting the transcription factor MITF in alpaca (Lama pacos) skin melanocytes. Domest Anim Endocrinol 38: 200-209, 2010. 